\title{
Are There Critical Points on the Boundaries of Singular Domains?
}

\author{
Michael R. Herman \\ Centre de Mathématiques, Ecole polytechnique, F-91128 Palaiseau Cedex, France
}

\begin{abstract}
Generalizing a result of E. Ghys, we prove a general theorem that implies that if a rational function $f$ of the Riemann sphere of degree $\geqq 2$ leaves invariant a singular domain $C$ (a disk or a ring) on which the rotation number of $f$ satisfies a diophantine condition, provided that on $\bar{C} f$ is injective, then each boundary component of $C$ contains critical point of $f$. The injectivity condition is always satisfied for singular disks associated to linearizable periodic elliptic points of $f(z)=z^{n}+a$, with $n \in \mathbb{N}, n \geqq 2$ and $a \in \mathbb{C}$. We also show that the singular disks, associated to periodic elliptic points of $f(z)=e^{a z}$ that satisfy a diophantine condition, are unbounded in $\mathbb{C}$. In the end of the paper, we give a survey of the theory of iteration of entire functions of $\mathbb{C}$.
\end{abstract}

\section{Introduction}

In 1920 , Fatou $[3, \S 30]$ showed that if a rational function $g$, of degree $\geqq 2$, left invariant a singular domain $C$, then the frontier (or boundary) of $C$ in $\bar{S}^{2}$ was contained in the $\omega$-limit set under $g$ of the set critical points of $g$ ( $C$ is either $\mathbb{C}$-diffeomorphic to $\mathbb{D}$ or to a ring). In 1942, Siegel [9] showed the existence of singular domains that are disks and for the existence of rings we refer to [6, I and VIII].

We propose to show in II.1, under the restrictions that the rotation number $\alpha$ of $g_{\mid C}$ satisfies a diophantine condition and $g_{\mid \bar{C}}$ is injective, then $g$ has at least one critical point in each component of $\operatorname{Fr}(C)$. This generalizes the partial result obtained by Ghys [4] who supposed that $\operatorname{Fr}(C)$ was a Jordan curve.

This result follows immediately from the main theorem (I.4). The proof of the main theorem was influenced by work of Ghys [4] who introduced diffeomorphisms of the circle, by using a form of conformal welding for special cases of Theorem 1 and related questions. The main ingredient to prove Theorem 1 is the fundamental theorem of [5, IX], as generalized by Yoccoz [10] (we use [10] to obtain rotation numbers that satisfy a diophantine condition and not the "unnatural" set of numbers of [5] that satisfy a condition A). The exact arithmetic for which the theorem of I.4 is true is an open question (see I.3). 
The question of proving the existence of a critical point of $g$ in each boundary component of $\operatorname{Fr}(C)$ (if $\alpha$ satisfies a diophantine condition) will have a positive answer if one can prove that, if $g^{\prime} \neq 0$ on $\operatorname{Fr}(C)$, then $g_{\mid \operatorname{Fr}(C)}$ is injective.

We have only been able to do this for the periodic elliptic points of the following polynomials:

$$
z \rightarrow z^{m}+a, \quad m \in \mathbb{N}, \quad m \geqq 2, \quad a \in \mathbb{C}
$$

We prove this in II.14. In II.15 we give other examples such that $g_{\mid \operatorname{Fr}(C)}$ has a critical value on $\operatorname{Fr}(C)$ and in II.16, we show that if $C$ is associated to a periodic elliptic orbit of $z \rightarrow e^{a z}$ (with a rotation number $\alpha$ that satisfies a diaphantine condition) then $C$ is unbounded in $\mathbb{C}$ (i.e. does not have compact closure in $\mathbb{C}$ ). The proofs use Sullivan's and Baker's non-wandering domain theorems ([S] and $\left[\mathrm{B}_{5}\right]$ ) as well as the classification III.6 to III.10.

In Appendix III we recall results of Fatou and Baker on the iteration of entire functions, and I would like to thank Baker for many discussions on this part. The examples of III.9 are original.

After I completed a proof of a weaker version of the main theorem, Peter Jones proposed considerable simplifications by suggesting the use, in I.7.1), of Carathéodory's kernel theorem and simplifying the structure of the proof of Theorem 1. Lemma 9 is due to him. The main theorem presented in I.4 is more general than the original one, the proof is simpler and the version presented should be considered, in part, joint work with Peter Jones. I would like to thank him for this as well as Etienne Ghys for communicating to me his results [4]. I would like to acknowledge many pleasant discussions with Noel Baker, Lennart Carleson, Adrien and Raphael Douady, Albert Fathi, Peter Jones and Jean Christophe Yoccoz.

I would like to thank Karin Lindberg for typing, with great care, an almost unreadable manuscript and acknowledge support from the N.S.F. during my visit at MSRI when the following paper was written. ${ }^{1}$

\section{The Main Theorem}

\section{Notations}

We denote the Riemann sphere by $\mathbb{S}^{2}=\mathbb{C} \cup\{\infty\}=\mathbb{P}\left(\mathbb{C}^{2}\right)$ with its canonical complex structure and topology.

If $A$ is a subset of $\mathbb{S}^{2}$ we denote by $\bar{A}$ the closure of $A$ in $\mathbb{S}^{2}$, by $\operatorname{Int}(A)$ the interior of $A$, and by $\operatorname{Fr}(A)$ the frontier (or boundary) of $A$ (i.e. $\bar{A} \cap \overline{\mathbb{S}^{2}-A \text { ). }}$

We put on $\mathbb{C}$ the metric $d(x, y)=|x-y|$.

If $L$ is compact contained in $\mathbb{C}$ we define, for $x \in \mathbb{C}$, the distance of $x$ to $L$ by:

$$
d(x, L)=\inf _{y \in L} d(x, y),
$$

and we have $\left|d\left(x_{1}, L\right)-d\left(x_{2}, L\right)\right| \leqq d(x, y)$.

1 After completing the following work, Lennart Carleson and Peter Jones, using a proof of Siegel's theorem by Jean-Christophe Yoccoz for $f_{\lambda}: z \rightarrow \lambda z+z^{2}$ and a e. $|\lambda|=1$, obtained a much simpler proof that $-\lambda / 2$ is on the boundary of the singular domain of $f_{\lambda}$ associated to 0 , for a.e. $|\lambda|=1$ 
We denote, for $\varepsilon>0$, the $\varepsilon$-neighborhood of $L$ by:

$$
V_{\varepsilon}(L)=\{x \in \mathbb{C} \mid d(x, L)<\varepsilon\} .
$$

We will use the following notations:

$$
\begin{gathered}
\mathbb{D}=\{z|| z \mid<1\} \quad \text { (for the disk), } \\
\mathbb{S}_{r}=\{z|| z \mid=r\} \quad \text { (for the circle of radius } r>0 \text { ), } \\
\mathbb{A}_{\varrho}=\{z \in \mathbb{C}|1<| z \mid<\varrho\}, \quad 1<\varrho \leqq+\infty \quad \text { (rings). }
\end{gathered}
$$

We recall that if $L$ is a compact connected subset of $\mathbb{S}, L \neq$ one point and $\emptyset \neq L \neq \mathbb{S}^{2}$, then every connected component of $\mathbb{S}^{2}-L$ is $\mathbb{C}$-diffeomorphic to the disk $\mathbb{D}$. Furthermore if $D \subset \mathbb{S}^{2}$ is a simply connected domain, then $\operatorname{Fr}(D)$ is connected.

\section{Rings}

We say that $A$, an open connected subset of $\mathbb{C}$ (or $\mathbb{S}^{2}$ ), is a ring (or annulus) if $A$ has the homotopy type of $\mathbb{S}_{1}$. It is elementary $[2, \S 330-331]$ to see that, if $A \neq \mathbb{S}^{2}-\{2$ points $\}$, then $A$ is $\mathbb{C}$-diffeomorphic to a unique $\mathbb{A}_{e}$.

Let $A$ be a ring, $A \neq \mathbb{S}^{2}$-\{2 points $\}, h: \mathbb{A}_{\varrho} \rightarrow A$ a $\mathbb{C}$-diffeomorphism ${ }^{2}$ and $f: A \rightarrow A$ a $\mathbb{C}$-diffeomorphism isotopic to the identity, then $h^{-1} \circ f \circ h: \mathbb{A}_{e} \rightarrow \mathbb{A}_{\rho}$ is of the form $z \rightarrow e^{2 \pi i \alpha} z, \alpha \in \mathbb{T}^{1}$ (see [8, 14-22]). We call $\alpha$ the rotation number of $f$ and $\pm \alpha$ is an invariant of topological conjugacy of $f$ (cf. [5, II]).

If $A \subset \mathbb{S}^{2}$ is a ring, then $\operatorname{Fr}(A)$ has 2 connected components (separated by a generator of the $\pi_{1}$ of $A$ ).

If $A \subset \mathbb{C}$ is a ring and $h: \mathbb{A}_{e} \rightarrow A$ is a $\mathbb{C}$-diffeomorphism, we denote by $L$ the component (called interior) of $\operatorname{Fr}(A)$ contained in the bounded component of $\mathbb{C}-h\left(\mathbb{S}_{\varrho_{1}}\right),\left(1<\varrho_{1}<\varrho\right)$.

If $U \subset C$ is open, we say that the function $f: U \rightarrow \mathbb{C}$ is univalent if $f$ is $\mathbb{C}$-analytic and injective. The mapping is also called conformal.

\section{Diffeomorphisms of the Circle}

Definition. We say that $\alpha \in \mathbb{R}(\bmod 1)$ satisfies a diophantine condition (and we denote this by $\alpha \in D C$ ) if there exists $\beta \geqq 0, \gamma>0$, such that for every $p / q \in \mathbb{Q}$, we have $|\alpha-(p / q)| \geqq \gamma q^{-(2+\beta)}$.

One has, if $n \in \mathbb{Z}^{*}$, then $\alpha \in D C \Leftrightarrow n \alpha \in D C$.

The main ingredient of the proof of the main theorem of this work, Sect. 4, is the following result:

Theorem. Let $f$ be an $\mathbb{R}$-analytic diffeomorphism of $\mathbb{S}_{1}$ of rotation number $\alpha \in D C$, then $f$ is $\mathbb{R}$-analytically conjugate on $\mathbb{S}_{1}$ to the rotation $z \rightarrow e^{2 \pi i \alpha} z$.

This theorem was first proved in [5] for $\alpha \in A$ (the set $A C D C$ and is of Haar measure 1) and using [5, A.2.4], it was generalized by Yoccoz [10] to $\alpha \in D C$ (see also [11]). It is an open question to know for what $\alpha \in \mathbb{T}^{1}-(\mathbb{Q} / \mathbb{Z})$ the above theorem is true. It is not totally unreasonable to conjecture that it is the case, if

2 Note. $\mathbb{C}$-diffeomorphism always means a surjection 
$\alpha \in \mathbb{R}-\mathbb{Q}$, and its convergents $\left(p_{n} / q_{n}\right)_{n \in \mathbb{N}}$ satisfy the following condition:

$$
\sum_{n \geqq 1} q_{n}^{-1} \log q_{n+1}<+\infty .
$$

Remark. In the proof of the main theorem, $\alpha \in D C$ is only needed (in the proof of Theorem 1) to apply the above theorem.

\section{The Main Theorem}

Let $A \subset \mathbb{C}$ be a ring, $A \neq \mathbb{C}$ - $\{1$ point $\}$ and $L$ the interior component of $\operatorname{Fr}(A)$. We suppose that, for some $\varepsilon>0$, the function $f: A \cup V_{\varepsilon}(L) \rightarrow \mathbb{C}$ is defined, holomorphic, and $f_{\mid A}$ is a $\mathbb{C}$-diffeomorphism isotopic to the identity of rotation number $\alpha$.

If $L$ is reduced to a point, then $A \cup L$ is $\mathbb{C}$-diffeomorphic to $\mathbb{D}$ (or $\mathbb{C}^{*}$ ) and $f$ is complex analytically conjugate to $z \rightarrow e^{-2 \pi i \alpha} z$. This is also the case if $\mathbb{S}^{2}-L$ is connected and $L$ then reduces to a point.

In the following we suppose that $L$ is not reduced to one point. Therefore we can find a $\mathbb{C}$-diffeomorphism $h: \mathbb{A}_{\varrho_{0}} \rightarrow A, 1<\varrho_{0} \leqq+\infty$, and we can suppose that $L=\bigcap_{1<\rho<\varrho_{0}} \overline{h\left(\mathbb{A}_{e}\right)}$.

Theorem. With the above hypothesis, if furthermore $f^{\prime} \neq 0$ on $L, f_{\mid L}$ is injective and $\alpha \in D C$, then $f$ leaves invariant a ring $\widetilde{A} \supset A \cup L$ such that $f_{\mid \tilde{A}}$ is a $\mathbb{C}$-diffeomorphism of rotation number $\alpha$ and h extends analytically to a neighborhood of $\mathbf{S}_{1}$ and $L$ is the analytic cricle $h\left(\mathbf{S}_{1}\right)$.

5.

To prove the main theorem we will need the following lemmas. We will suppose all the hypotheses of 4 satisfied, though in Lemma 1 the hypotheses $f^{\prime} \neq 0$ on $L$ and $f_{\mid L}$ injective are not used, and $\alpha \in D C$ is only used to prove Theorem 1.

Lemma 1. $f(L)=L$.

Proof. $f(L) \subset L$ (since $f_{\mid A}$ is isotopic to the identity, $f_{\mid A \cup L}$ is continuous and $f_{\mid A}$ is a homeomorphism).

$f(L)=L$. Let $y \in L$ and $\left(y_{i}\right)_{i \in \mathbb{N}}$ be a sequence of points of $A$ converging to $y$. Let $\left(x_{i^{\prime}}\right)_{i^{\prime}}$ be a convergent subsequence of $\left(f^{-1}\left(y_{i}\right)\right)_{i}$ to $(x \in L)$. As $f_{\mid A \cup L}$ is continuous, $f(x)=y$.

We will now suppose that $f^{\prime} \neq 0$ on $L$ and that $f_{\mid L}$ is injective.

Since $f(L)=L, f_{\mid A \cup L}$ is injective.

Lemma 3. There exists $\delta>0$, such that $f_{\mid A \cup V_{\delta}(L)}$ is univalent.

Proof. By the implicit function theorem, $f$ is locally injective on $V_{\varepsilon_{1}}(L)$, for some $\varepsilon_{1}$ $>0$ : if $x_{1}$ and $x_{2} \in V_{\varepsilon_{1}}(L)$ and $f\left(x_{1}\right)=f\left(x_{2}\right)$, then $d\left(x_{1}, x_{2}\right) \geqq \frac{1}{4} \varepsilon_{1}$.

If the lemma is false, then for every $\delta>0$, there exists $x_{1}(\delta) \neq x_{2}(\delta)$ with $f\left(x_{1}(\delta)\right)$ $=f\left(x_{2}(\delta)\right)$, and $d\left(x_{j}(\delta), L\right)<\delta,(j=1,2)$. Letting $\delta_{i} \rightarrow 0$ for converging subsequences $\left(x_{1}\left(\delta_{i}\right)\right)_{i}$ and $\left(x_{2}\left(\delta_{i}\right)\right)_{i}$, we contradict the fact that $f_{\mid L}$ is injective.

Remark 1. - As $f_{\mid A \cup V_{\varepsilon}(L)}$ is continuous, by Lemma 1, given $\eta_{1}>0$, there exists $\eta_{2}>0$, such that

$$
f\left(V_{\eta_{2}}(L)\right) \subset V_{\eta_{1}}(L) .
$$


- There exists $\delta_{1}>0$, such that $\mathrm{f}_{\mid A \cup V_{\delta_{1}}(L)}$ is univalent (this follows by the above remark and by the same argument as in Lemma 3 applied to $\left.\overline{h\left(\mathbb{A}_{\varrho_{1}}\right)}, 1<\varrho_{1}<\varrho_{0}\right)$. We denote by $f_{\mid A \cup f\left(V_{\delta_{1}}(L)\right)}^{-1}$ its inverse function (that extends analytically $\left(f_{\mid A}\right)^{-1}$ $\left.\equiv f_{A}^{-1}\right)$.

- One obtains: given $n \geqq 1$, one can find $\delta_{n}>0$, such that $f_{\mid A \cup V_{\delta_{n}}(L)}^{ \pm n}$ are well defined and univalent.

Notations. Let $\bigcup_{i \in Q} C_{i}=\mathbf{S}^{2}-L$, the $\left(C_{i}\right)_{i \in Q}$ being the connected components and $Q$ a set of indices. We will suppose that $\infty \in Q$ and $C_{\infty}$ denotes the connected component of $\boldsymbol{S}^{2}-L$ containing $\infty$.

Each $C_{i}$ is $\mathbb{C}$-diffeomorphic to $\mathbb{D}$. Let $Q_{i}$ be the set of $i \in Q$ such that

$$
C_{i} \subset V_{\delta}(L) \text { ( } \delta \text { being the number given by Lemma } 3 \text { ). }
$$

We will allow the possibility that $Q_{1}=\emptyset$. Let $Q_{2}=Q-Q_{1}$.

As $L$ is not reduced to one point, $\mathbb{S}^{2}-L$ is not connected.

Lemma 4. The set $Q_{2}$ is finite.

Proof. Otherwise there exists an infinite sequence $\left(x_{i}\right)_{i \in Q_{2}}$ of points, with $x_{i} \in C_{i}$ if $i \in Q_{2}$ and satisfying $d\left(x_{i}, L\right) \geqq \delta$. Let $y$ be a limit point of the sequence $\left(x_{i}\right)_{i}$. As $y \notin L$, $\mathrm{y} \in \mathrm{C}_{i_{0}}, i_{0} \in Q$, we arrive to a contradiction if $Q_{2}$ is infinite.

Lemma 5. Let $D \subset \mathbb{C}$ be a bounded simply connected domain and $l: \mathbb{D} \rightarrow D$ a $\mathbb{C}$-diffeomorphism. Given $\varepsilon>0$, we can find $r_{0}\left(0<r_{0}<1\right)$ such that, if $r_{0}<r<1$, then

$$
l\left(\mathbb{S}_{r}\right) \subset V_{\varepsilon}(\operatorname{Fr}(D)) .
$$

Proof. As the set $V=\{x \in \bar{D} \mid d(x, \operatorname{Fr}(D)) \geqq \varepsilon\}$ is compact and contained in $D$, the compact set $l^{-1}(V)$ is compact contained in $\mathbb{D}$ and the lemma follows easily.

Remark 2. A similar lemma is true for a ring $A \subset \mathbb{C}, A=h\left(\mathbb{A}_{\varrho}\right)$, considering the interior component $L$ on $\operatorname{Fr}(A)$.

- If $r \rightarrow 1$, then given $\varepsilon>0$, we can find $r_{1}\left(0<r_{1}<1\right)$ such that, for $r_{1}<r<1$,

$$
\operatorname{Fr}(D) \subset V_{\varepsilon}\left(l\left(\mathbf{S}_{r}\right)\right) \text {. }
$$

(This easily follows from [7, Corollary 9.3, p. 277] by considering a finite covering of $\operatorname{Fr}(D)$ by disks of radii $\varepsilon / 4$ and by choosing an accessible point in each disk.)

Lemma 6. If $Q_{1} \neq \emptyset$, let $C_{i}$, with $i \in Q_{1}$, (i.e. $f_{\mid C_{i}}$ is holomorphic and univalent), then $f\left(C_{i}\right)=C_{j}$ for some (unique) $j \in Q$ (and $f$ is a $\mathbb{C}$-diffeomorphism of $C_{i}$ onto $C_{j}$ ).

Proof. The set $f\left(C_{i}\right)$ is open and connected and does not intersect $L$, hence $f\left(C_{i}\right) \subset C_{j}$ with $j \in Q$. But $f\left(C_{i}\right) \cap C_{j}=f\left(\overline{C_{j}}\right) \cap C_{j}$ [since $\left.f\left(\operatorname{Fr}\left(C_{i}\right)\right) \subset L\right]$, hence $f\left(C_{i}\right) \cap C_{j}$ is closed (and open) in the connected set $C_{j}$.

Remark 3. Using Lemma 5 and Remark 1, we see that, for $i \in \mathbb{Q}_{2}, f$ sends univalently, a small enough neighborhood of $\operatorname{Fr}\left(C_{i}\right)$ in $C_{i}$ (that is a ring) onto a ring that is a neighborhood of $\operatorname{Fr}\left(C_{j}\right)$ in $C_{j},(j \in Q)$. [A neighborhood $N$ of $\operatorname{Fr}\left(C_{i}\right)$ in $C_{i}$ means that $N$ contains $V_{\varepsilon}\left(\operatorname{Fr}\left(C_{i}\right)\right) \cap C_{i}$, for some $\varepsilon>0$.] 
Lemma 7. Let $0<r_{0}$ and $g:\left\{r_{0}<|z|<1\right\} \rightarrow \mathbb{D}-\{0\}$ be a univalent function onto the ring bounded by $g\left(\mathbb{S}_{r_{0}}\right)$ and $\mathbb{S}_{1}$, homotopic to the identity. Then $g$ extends on $\mathbf{S}_{1}$ to an orientation preserving $\mathbb{R}$-analytic diffeomorphism of $\mathbf{S}_{1}$.

Proof. Since, if $r_{0}<r_{1}<1$ then $\bigcap_{r_{1}<r<1} \overline{g(\{r<|z|<1\})}=\mathbb{S}_{1}$, by Schwarz's reflexion principle $\left[8,11\right.$.7] $g$ extends to an $\mathbb{R}$-analytic mapping from $\mathbb{S}_{1}$ to $\mathbb{S}_{1}$. By replacing $g$ by $g^{-1}$ the lemma follows.

Lemma 8. For $i \in Q_{2}$ we can extend $f$ to a $C^{\infty}$-orientation preserving diffeomorphism $F_{i}$ of $C_{i}$ onto $C_{j}$, and equal to $f$ on a neighborhood of $\operatorname{Fr}\left(C_{i}\right)$ in $C_{i}$.

Proof. This follows immediately from the isotopy extention theorem. We can also see this in the following way. Let $\varphi_{i}: \mathbb{D} \rightarrow C_{i}$ and $\varphi_{j}: \mathbb{D} \rightarrow C_{j}$ be conformal maps (onto). Using Remark 3 (and 1), we let

$$
g=\varphi_{j}^{-1} \circ f \circ \varphi_{i}:\left\{r_{0}<|z|<1\right\} \rightarrow \mathbb{D} \text { for some } r_{0}>0 .
$$

By Lemma 7, if $r \rightarrow 1, g\left(\mathbb{S}_{r}\right)$ is $C^{\infty}$ close to $\mathbb{S}_{1}$. By considering the radial projection from 0 , one easily constructs a $C^{\infty}$-diffeomorphism $H$ of $\mathbb{D}$, equal to the identity in a neighborhood of $\mathbb{S}_{1}$ in $\mathbb{D}$, and such that $H \circ g\left(\mathbb{S}_{r_{1}}\right)=\mathbb{S}_{r_{1}}$ for some $r_{0}<r_{1}<1$. The rest of the construction is immediate.

We define $F: \mathbb{S}^{2} \rightarrow \mathbb{S}^{2}$ of class $C^{\infty}$ (see Lemma 4) by:

$$
\begin{gathered}
F(x)=F_{i}(x), \quad \text { if } \quad x \in C_{i}, i \in Q_{2} ; \\
F(x)=f(x), \quad \text { if } \quad x \notin \bigcup_{i \in Q_{2}} C_{i} .
\end{gathered}
$$

$F$ is a $C^{\infty}$-orientation preserving diffeomorphism of $\mathbb{S}^{2}$ (since $f_{\mid V_{\delta}(L)}$ is univalent, or also since $F$ is an immersion of $\boldsymbol{S}^{2}$ ).

We will make the following conventions on the extention $F$ (and we will use that same letter $F$ for possibly different extensions).

Convention 1. On $C_{\infty}$, we extend $F$ such that $F$ is $C^{\infty}$-conjugate to $z \rightarrow e^{-2 \pi i \alpha} z$ (on D) and $F(z)=e^{2 \pi i \alpha} z$ in a neighborhood of $\infty$ (i.e. if $|z|$ is big enough) (it is enough to extend the diffeomorphism $h$ of Sect. 4 as in the proof of Lemma 8).

Convention 2. If $i \in Q_{2}-\{\infty\}$ and $C_{i}$ is a periodic component by $F$ of period $q$ [i.e. $\left.F^{q}\left(C_{i}\right)=C_{i}\right]$ then we will suppose that $F$ has a periodic point $p_{i} \in C_{i}$ of period $q$.

Convention 3. If $i \in Q_{2}-\infty$ and if $C_{i}$ is a periodic component by $F$ of period $q$, such that $f^{q}$ leaves invariant arbitrarily small neighborhoods of $\operatorname{Fr}\left(C_{i}\right)$ in $C_{i}$ that are rings, then we extend $F$ on $\left(f^{k}\left(C_{i}\right)\right)_{0 \leqq k \leqq q-1}$ in such a manner that $F_{\mid C_{i}}^{q}$ is $C^{\infty}$-conjugate to a rotation (this is possible using 2 and a similar construction on the one in the proof of Lemma 8).

6.

For the proof of the main theorem we need the following intermediate theorem.

Theorem 1. Let $C_{i}$ be periodic component by $F$ of period $q \geqq 1$, with $i \neq \infty$ and $i \in Q_{2}$, then $f^{q}$ leaves invariant a ring $A_{1}$ that is a neighborhood of $\operatorname{Fr}\left(C_{i}\right)$ in $C_{i}$ and such that $f_{\mid A_{1}}^{q}$ is a $\mathbb{C}$-diffeomorphism, isotopic to the identity and of rotation number $q \alpha$. 
Proof of Theorem 1. For $1<\varrho<\varrho_{0}$, let $\Sigma_{\varrho}=h\left(\mathbb{S}_{\varrho}\right)$, let $D_{\varrho}$ be the bounded connected component of $\mathbb{C}-\Sigma_{\varrho}$ and $g_{\varrho}$ the conformal representation of $\mathbb{D}$ onto $D_{\varrho}$ such that: $g_{\rho}^{\prime}(0) \in \mathbb{R}_{+}^{*}, g_{\varrho}(0)=p_{i}$ is the fixed point of $F^{q} c_{i^{*}}$. (See Convention 2, $p_{i}$ does not depend on $\varrho$.)

1) As $C_{i}$ is the connected component containing $p_{i}$ in $\operatorname{Int}\left(\bigcap_{\varrho>1} D_{\varrho}\right)$, by Carathéodory's kernel theorem [7, pp. 28-31], if $\varrho \rightarrow 1, g_{\varrho} \rightarrow g_{1}$ in the compact open topology on $\mathbb{D}$ and $g_{1}: \mathbb{D} \rightarrow C_{i}$ is the unique conformal representation of $\mathbb{D}$ onto $C_{i}$ such that $g_{1}(0)=p_{i}$ and $g_{1}^{\prime}(0) \in \mathbb{R}_{+}^{*}$.

2) For $1<\varrho<\varrho_{0}, f_{\varrho}=g_{\varrho}^{-1} \circ F^{q} \circ g_{\varrho}$ is a $C^{\infty}$-diffeomorphism of $\overline{\mathbb{D}}$, analytic in a neighborhood of $\mathbb{S}_{1}$ in $\mathbb{C}\left[g_{e}\right.$ is a nalytic in a neighborhood of $\mathbb{S}_{1}$ since $g_{\varrho}\left(\mathbf{S}_{1}\right)=\Sigma_{\varrho}$ is an $\mathbb{R}$-analytic submanifold of $\mathbb{C}[2$, Sect. 349]].

The $\mathbb{R}$-analytic diffeomorphism $f_{e \mid \mathbb{S}_{1}}$ is $\mathbb{R}$-analytically conjugate on $\mathbb{S}_{\mathbf{1}}$ (by $\left.g_{\ell}^{-1} \circ h\right)$ to $z \rightarrow e^{2 \pi i q \alpha} z$.

3) The diffeomorphisms $f_{\varrho}\left(1<\varrho<\varrho_{0}\right)$ are $K$-quasiconformal (q.c.) for some finite $K$ independent of $\varrho$ (since $F^{q}$ is $K$-q.c. and $g_{\varrho}$ is conformal).

4) By Mori's theorem (see for example [1, p. 47]), since $f_{\varrho}(0)=0$, we have:

$$
\left(\frac{1}{16} d(x, y)\right)^{K} \leqq d\left(f_{\varrho}(x), f_{\varrho}(y)\right) \leqq 16(d(x, y))^{1 / K} \quad \text { for } \quad x, y \in \overline{\mathbb{D}} .
$$

By Ascoli's theorem, a subsequence $\left(f_{\varrho_{i}}\right)_{i}, \varrho_{i} \rightarrow 1,\left(\varrho_{i}>1\right)$ converges uniformly on $\overline{\mathbb{D}}$ to a $K$-q.c. homeomorphism $f_{1}$ of $\mathbb{D}$ [since the rotation number is continuous in the uniform $\left(C^{0}\right.$-)topology, the rotation number of $f_{1 \mid \mathbb{s}_{1}}$ is $q \alpha$ (see $\left.\left.[5, \mathrm{II}]\right)\right]$.

5) Writing

$$
F^{q} \circ g_{e_{i}}=g_{e_{i}} \circ f_{e_{i}}
$$

and using 4), we can let $\varrho_{i} \rightarrow 1$ and take limits in the compact open topology on $\mathbb{D}$, and therefore

$$
g_{1}^{-1} \circ F^{q} \circ g_{1}=f \text {, on } \mathbb{D} \text {. }
$$

At this point the reader should also consult [4]. It follows (cf. Remark 3) that $f_{1}$ is holomorphic on a neighborhood of $\mathbf{S}_{1}$ in $\mathbb{D}$, and by Schwarz's reflexion principle we conclude that the mapping $f_{1 \mid \mathbb{S}_{1}}$ is $\mathbb{R}$-analytic. If we use at the same time $F^{-q}$ we deduce (see also Remark 3 and Lemma 7) that $f_{1 \mid \mathbb{s}_{1}}$ is an $\mathbb{R}$-analytic diffeomorphism (orientation preserving) of rotation number $q \alpha \in D C$.

By Sect. 3, $f_{1 \mid \mathbb{S}_{1}}=h \circ R_{q \alpha} \circ h^{-1}, h$ being an $\mathbb{R}$-analytic (orientation preserving) diffeomorphism of $\mathbb{S}_{1}$ and $R_{q \alpha}: z \in \mathbb{S}_{1} \rightarrow e^{2 \pi i q \alpha} z \in \mathbb{S}_{1}$. Theorem 1 now follows by complexifying $h$ and considering $g_{1}^{-1} \circ f_{1} \circ g_{1}$ (with 1 ).

Using Theorem 1 we consider a $C^{\infty}$-diffeomorphism $F$ extending $f$ that satisfies the Conventions 1 and 3.

Lemma 9 (Peter Jones). The sequence $\left(F^{n}\right)_{n \in \mathbb{Z}}$ is uniformly equicontinuous on $\mathbb{S}^{2}$. Proof. a) Let us show first that $F^{n}$ is $K$-quasiconformal for some finite $K$ independent of $n$ (we refer the reader to [1, Chap. I] for definitions and elementary properties):

1) If $F_{\mid C_{i}}^{n}$ is holomorphic, then $F_{\mid C_{i}}^{n}$ is 1-q.c. This is also the case of $F_{\mid L}^{n}$. 
2) If $C_{i}$ is wandering [i.e. $\left(F^{n}\left(C_{i}\right)\right)_{n \in \mathbb{Z}}$ are mutually disjoint] then $F$ is holomorphic and univalent on $F^{j}\left(C_{i}\right), 0 \leqq j \leqq n$, except for a fixed finite number of $j$ 's [at most $\left.p=\operatorname{Card}\left(Q_{2}\right)\right]$. Since $F$ is a $C^{\infty}$-diffeomorphism of $\mathbb{S}^{2}, F$ is $K_{1}$-q.c. and $F_{\mid C_{i}}^{n}$ is $K_{1}^{p}$-q.c.

3) If $C_{i}$ is a periodic component [i.e. $F^{q}\left(C_{i}\right)=C_{i}$ ], and, if the whole orbit of $C_{i}$ by $F$ is indexed by $Q_{1}$, then $F_{\mid C C_{i}}^{n}$ is 1 -q.c.

4) By Lemma 4, we are left with at most a finite number of periodic components of period $q_{j}$ indexed by a subset of $Q_{2}$. This case is taken care of if we use Convention 3. (For $C_{\infty}$ we can use, for example, Convention 1.) This proves a).

b) By Convention $1, e^{-2 \pi i n a} F^{n}$ is the identity on $\{|z|>R\}$ for some large $R>1$. Let $H$ be a Möbius transformation such that $H(0)=2 R$ and $H\left(\mathbb{S}_{1}\right)=\mathbb{S}_{3 R}$. Applying Mori's theorem [1] to $H^{-1} \circ\left(e^{-2 \pi i n \alpha} F^{n}\right) \circ H$ on $\mathbb{D}$, the lemma follows from a), by conjugating back by $H$. [One even obtains that the sequence $\left(F^{n}\right)_{n \in \mathbb{Z}}$ is uniformly Hölder of exponent $1 / K$ for the metric $d_{1}$ on $\mathbb{S}^{2} \subset \mathbb{R}^{3}$ induced by the standard metric of $\mathbb{R}^{3}$.]

Corollary 1. All the components $C_{i}$ are periodic.

Proof. Let $C_{i}$ be a wandering component, $x \in C_{i}, D$ a small disk centered at $x$ contained in $C_{i}$. By Lemma 9, there exists $\eta>0$ such that each $F^{n}(D), n \in \mathbb{Z}$, contains a disk of center $F^{n}(x)$ and of radius $\eta$. As all the $\left(F^{n}(D)\right)_{n \in \mathbb{Z}}$ are mutually disjoint [and $\left.F\left(C_{\infty}\right)=C_{\infty}\right]$ this contradicts the fact that the sum of the areas of the $F^{n}(D)$ is finite.

Corollary 2. $f$ has no periodic point on $L$.

Proof. If $x \in L$, satisfies $f^{q}(x)=x$, then by Lemma $9,\left|\left(f^{q}\right)^{\prime}(x)\right|=1$ and on a neighborhood $D_{x}$ of $x, f^{q}$ would be conjugate to a rotation on $D_{x}$. As $D_{x} \cap A \neq \emptyset$ and $\alpha \notin \mathbb{Q} / \mathbb{Z}$, this is only possible if $L=1$ point.

Proof of the Main Theorem. By Corollary 1 and Theorem 1 applied to $C_{i}$, $i \in Q_{2}-\{\infty\}, f$ leaves invariant an open connected set $U$ containing $A \cup L$ such that $\left.f\right|_{U}$ is a $\mathbb{C}$-diffeomorphism. The closure of $\left(\left.f^{n}\right|_{U}\right)_{n \in \mathbb{Z}}$ for the compact open topology on $U$, is a compact group $G$ of complex analytic diffeomorphisms. We have $G \cong \mathbb{T}^{1}$, since $\alpha \notin \mathbb{Q} / \mathbb{Z}$ and the action of $G$ on $U$ extends the action of $\mathbb{T}^{1}$ on $A$ obtained by conjugating by the diffeomorphism $h$, the canonical action of $\mathbb{T}^{1}$ on $\mathbb{A}_{\varrho_{0}}: t \rightarrow R_{t}$, $R_{t}(z)=e^{2 \pi i t_{z}}$. (In fact, the action of $G$ on $U$ is $\mathbb{R}$-analytic but this is a consequence of what follows.)

We conclude that $L$ is a Jordan curve or a point (cf. Corollary 2), and as $L \neq 1$ point, $f_{\mid L}$ is not the identity. Using the action of $G$ we conclude that $f$ leaves invariant a ring containing $A \cup L$. The theorem follows from 2 and the argument [2, Sect. 350] using Schwarz's reflexion principle to extend $h$ analytically in a neighborhood of $\mathbb{S}_{1}$.

\section{Examples of Application of the Main Theorem}

\section{Rational Functions}

Let $g: \mathbb{S}^{2} \rightarrow \mathbb{S}^{2}$ be a rational function of degree $d \geqq 2, J(g)$ its Julia set and $C$ an 
invariant singular component of $\mathbb{S}^{2}-J(g)$, or equivalently $g_{\mid C}$ is a $\mathbb{C}$-diffeomorphism of $C[3$, Sect. 30]).

By [6], $C$ is $\mathbb{C}$-diffeomorphic to $\mathbb{D}$ (a disk) or to a unique $\mathbb{A}_{o}, 1<\varrho<+\infty$ (a ring) and $g_{\mid C}$ is complex analytically conjugate to $z \rightarrow e^{2 \pi i \alpha} z, \alpha \in \mathbb{T}^{\mathbf{1}}-(\mathbb{Q} / \mathbb{Z})$.

The existence of singular components that are disks follows from Siegel's theorem [9] and for the existence of rings, see [6]. (Siegel's theorem is a consequence of the appropriate local existence theorem of rings [6, VIII.6 and 12] and by Brjuno's results [BJ] Siegel's theorem is also valid for certain Liouville numbers, see $[6,1.4]$ for other references.)

If $\alpha \in D C$ and $g_{\mid \bar{C}}$ is injective, then in every boundary component of $C$ (one for a disk, 2 for a ring), there exists at least one critical point of $g$ [a critical point $c$ means $g^{\prime}(c)=0$ in $\mathbb{C}$-charts of $\mathbb{S}^{2}$ ]. If this were not the case we would contradict the maximality of $C$ [i.e. $\operatorname{Fr}(C) \subset J(g)$ ], by using I.4 (after conjugating $g$ by an appropriate Möbius transformation).

\section{Examples}

From what we see above this reduces the question of the existence of critical points of $g$ in the boundaries of $C$ to showing that $g_{\mid \bar{C}}$ is injective [if we suppose that $f$ has no critical point on $\operatorname{Fr}(C)]$.

We have only been able to show this for the periodic elliptic points of multiplier $e^{2 \pi i \alpha}, \alpha \in D C$, of the following polynomials:

$$
f(z)=z^{m}+a, \quad m \in \mathbb{N}, \quad m \geqq 2, \quad a \in \mathbb{C} .
$$

For the proof of this we refer the reader to 14 .

Using the above examples and Douady-Hubbard's theory on polynomial like mappings [D], one can construct many other examples of entire functions $f$ that have a periodic disk $C$ of rotation number $\alpha \in D C$ (i.e. $\alpha$ is the rotation number $f^{q}$ where $q$ is the period of $C$ ) and such that there exists a critical point of $f$ in the boundary of the one of the disks $f^{j}(C), j \in \mathbb{N}$.

In 15 , we will give other examples of rational functions $f$ that have a periodic disk $C$ of rotation number $\alpha \in D C$ and periodic $q$, but we will only show that $f^{q}$ has a critical value in the boundary of $C$.

\section{Entire Functions}

Let $g$ be an entire function, such that $g(0)=0$ and $g^{\prime}(0)=e^{2 \pi i \alpha}=\lambda$ with $\alpha \in D C$. Let $C$ be the component of $\mathbb{C}-J(g)$ containing $0(C \neq \emptyset$ by Siegel's theorem $)$ and by III.10 $C$ is $\mathbb{C}$-diffeomorphic to $\mathbb{D}$. By the same argument as in 1 , if $C$ has compact closure in $\mathbb{C}$ and if $g_{\mid \bar{C}}$ is injective, than $g$ has a critical point on $\operatorname{Fr}(C)$.

Remark. By Carathéodory's theorem [7, p. 279], if $\infty \in \bar{C}$, then $\operatorname{Fr}(C) \subset \mathbb{S}^{2}$ is neither a Jordan curve nor locally connected (if $g$ is not polynomial of degree 1):

Let $\left(y_{i}\right)_{i \in \mathbb{N}} \subset C$ be a sequence tending to $\infty$. Then the sequence $\left(\left(g_{\mid C}\right)^{-n}\left(y_{i}\right)\right)_{i \in \mathbb{N}}$ ( $n \geqq 1$ fixed) tends to $\infty$ and therefore a $\mathbb{C}$-diffeomorphism $l: \mathbb{D} \rightarrow C, l(0)=0$, cannot extend continuously to $\mathbb{S}_{1}\left[\right.$ since $l^{-1} \circ g \circ l(z)=\lambda z$, for $|z|<1$ and $\left.\alpha \in \mathbb{R}-\mathbb{Q}\right]$. By the above argument it is not difficult to see that the impression of every prime end of $C$ contains $\infty$. 


\section{Example}

We will show in 16 , that for $f(z)=e^{a z}, a \neq 0$, every maximal periodic disk of $f$ of rotation number $\alpha \in D C$ has non-compact closure in $\mathbb{C}$.

\section{Functions on $\mathbb{D}$}

Let $g: \mathbb{D} \rightarrow \mathbb{C}$ be a holomorphic function that does not extend beyond $\mathbb{D}$. We suppose that $g(0)=0, g^{\prime}(0)=e^{2 \pi i \alpha}=\lambda$ with $\alpha \in D C$. Let $C$ be the maximal $g$-invariant open connected set containing 0 . By Siegel's theorem $C \neq \emptyset$, and it is not difficult to see that, $C \subset \mathbb{D}$, is $\mathbb{C}$-diffeomorphic to $\mathbb{D}$ and that $g_{\mid C}$ is $\mathbb{C}$-conjugate to $z \rightarrow \lambda z$. $\operatorname{Fr}(C)$.

By I.4, if $\bar{C} \subset \mathbb{D}$ and $g_{\mid \bar{C}}$ is injective, then there exists a critical point of $g$ on 6.

Let $f: \mathbb{C} \rightarrow \mathbb{C}$ be an entire function and we suppose that $f$ is not a polynomial of degree 0 or 1 (but $f$ can be a polynomial).

We denote by $J(f)$ the Fatou-Julia set of $f\left(\mathrm{cf}\right.$. III). We suppose that $z_{0} \in \mathbb{C}$ is a periodic point of period $q$. Furthermore we suppose that $z_{0} \notin J(f)$ and that $\left(f^{q}\right)^{\prime}\left(z_{0}\right)=e^{2 \pi i \alpha}, \alpha \in \mathbb{R}$. It follows that $\alpha \in \mathbb{R}-\mathbb{Q}$. We will suppose in 14 and 16 that $\alpha \in D C$, but we will not need this until then. Let $C$ be the component of $\mathbb{C}-J(f)$ containing $z_{0}$. By III.10, $C$ is $\mathbb{C}$-diffeomorphic to $\mathbb{D}$.

We suppose that $C$ has compact closure in $\mathbb{C}$ (i.e. is bounded in $\mathbb{C}$; this is necessarily the case if $f$ is a polynomial of degree $d \geqq 2$ ). It follows that for all $j \in \mathbb{N}$ $f^{j}(C)$ has compact closure in $\mathbb{C}$.

7.

As $C$ is simply connected, $\operatorname{Fr}(C)$ is connected. Let $\mathbb{S}^{2}-\operatorname{Fr}(C)=C \amalg C_{\infty} \coprod_{i \in I} C_{i}$ be the connected components, where $\infty \in C_{\infty}$ and $I$ is a set of indices at most countable (and possibly empty).

Each component $C, C_{\infty}$ or $C_{i}$ is simply connected.

We denote the "filled up" compact set of $C$ by

$$
\hat{C}=\mathbb{S}^{2}-C_{\infty} \text {. }
$$

The compact set $\hat{C}$ is connected and has a fundamental system of neighborhoods that are connected and simply connected. We have $\hat{C} \supset C \cup\left(\bigcup_{i \in I} C_{i}\right)$. If we suppose $I \neq \emptyset$ then each $C_{i}, i \in I$, has compact closure. We have:

8.

Lemma 10. Each $C_{i}, i \in I$, is a component of $\mathbb{C}-J(f)$.

Proof. It is enough to see this for $g=f^{k}$ (cf. III.2.b). By Lemma $1, g(\operatorname{Fr}(C))=\operatorname{Fr}(C)$, and since $\operatorname{Fr}\left(C_{i}\right) \subset \operatorname{Fr}(C)$ by the maximum modulus principle the family $\left(g_{\mid C_{i}}^{n}\right)_{n \geqq 1}$ is normal since bounded. It follows that $C_{i} \subset \mathbb{C}-J(g)$ but as $\operatorname{Fr}\left(C_{i}\right) \subset \operatorname{Fr}(C) \subset J(g)$ it easily follows that $C_{i}$ is a component of $\mathbb{C}-J(g)$. 
9.

Lemma 11. We have $\operatorname{Fr}(C)=\operatorname{Fr}\left(C_{\infty}\right)$.

Proof. Since $\operatorname{Fr}\left(C_{\infty}\right) \subset \operatorname{Fr}(C)$, if we suppose $y \in\left(\operatorname{Fr}(C)-\operatorname{Fr}\left(C_{\infty}\right)\right) \neq \emptyset$, then we can find a disk $D \ni y$ such that $\left(f_{\mid D}^{n}\right)_{n \geqq 1}$ is a normal family since it is bounded, and this contradicts $y \in J(f)$.

10.

Remark. The above lemma does not imply a priori, that $C_{i}=\emptyset$; this fact can be seen using Wada lakes and similar examples [HY].

11.

By the same argument as in Lemma 1 [the only things one uses is that $f: f^{j}(C)$ $\rightarrow f^{j+1}(C), j \in \mathbb{N}$, is a homeomorphism and $f^{j}(C)$ has compact closure in $\left.\mathbb{C}\right]$ we have

$$
\operatorname{Fr}\left(f^{j+1}(C)\right)=f\left(\operatorname{Fr}\left(f^{j}(C)\right) \subset J(f) .\right.
$$

We deduce from this and Lemma 10 that

$$
\left.f \widehat{\left(f^{j}(C)\right.}\right) \subset \widehat{f^{j+1}(C)} \text {, }
$$

where $\widehat{f^{j}(C)}$ is defined in a similar way to $\hat{C}$.

For the definition of singular values we refer the reader to III.3.

12. Lemma 12. If $f$ has no singular values in a neighborhood of $\bigcup_{0 \leqq j \leqq q-1} \widehat{f^{j}(C)}$ then, for
every $0 \leqq j \leqq q$,

$$
f_{\mid \operatorname{Fr}\left(f^{j}(C)\right)}: \operatorname{Fr}\left(f^{j}(C)\right) \rightarrow \operatorname{Fr}\left(f^{j+1}(C)\right)
$$

is a homeomorphism.

Proof. Let $V$ be a connected and simply connected neighborhood of $\widehat{f^{j+1}(C)}$ such that $f$ has no singular value in $V$. Since $f_{\mid f^{-1}(V)}: f^{-1}(V) \rightarrow V$ is a trivial covering ${ }^{3}$, the C-diffeomorphism

$$
\left(f_{\mid f^{j+1}(C)}\right)^{-1}: f^{j+1}(C) \rightarrow f^{j}(C),
$$

extending continuously to a continuous map $h$ from $\overline{f^{j+1}(C)}$ into $\overline{f^{j}(C)}$, and the lemma follows from:

$$
f \circ h(z)=z, \quad \text { if } \quad z \in f^{j+1}(C)
$$

and

$$
h \circ f(z)=z, \text { if } \quad z \in f^{j}(C)
$$

3 We use the word covering in the sense of revêtement (in French) 
13.

Lemma 13. We suppose that:

a) No component of $\mathbb{C}-J(f)$ is wandering.

b) If we denote by $S(f)$ the set of singular values of $f$, then there exists $s_{0} \in S(f)$ such that
i) $\lim _{n \rightarrow+\infty} f^{n}(s)=\infty$ for every $s \in S(f)-\left\{s_{0}\right\}$,
ii) $\inf _{s \in S(f)-\left\{s_{0}\right\}} \inf _{n \in \mathbb{N}} d\left(f^{n}(s), \operatorname{Fr}(C)\right)>0$

[i) implies ii) if $S(f)$ is finite].

c) For every $0 \leqq j \leqq q-1, s_{0} \notin \operatorname{Fr}\left(f^{j}(C)\right)$.

Then

$$
g_{\mid \operatorname{Fr}(C)}: \operatorname{Fr}(C) \rightarrow \operatorname{Fr}(C)
$$

is injective.

Proof. By Fatou's result the set $\left.\overline{\bigcup_{n \geq 0} f^{n}(S(f)}\right)$ contains $F=\bigcup_{j \geqq 0} \operatorname{Fr}\left(f^{j}(C)\right)$, see III.10. By b) it follows that $\Omega=\left\{\frac{n \geqq 0}{f^{n}\left(s_{0}\right), n \geqq 0}\right\}$ contains $F$. Let us prove that $s_{0} \in J(f)$. If $s_{0} \notin J(f)$, then using a) and the classification III. 6 to 10 , we would contradict $\Omega \supset F$ and hence $s_{0} \in J(f)$. Now, by c) and Lemma 10, all the hypothesis of Lemma 12 are satisfied and Lemma 13 then follows from Lemma 12.

Remark. If a) and b) are true, then we always have $s_{0} \in J(f)$.

In the following examples condition a) of Lemma 13 is automatically satisfied by Sullivan's theorem and Baker's theorem, cf. III.7. We will also need the full strength of I.4, for in 7 we could have $\operatorname{Fr}\left(C_{i}\right)=\operatorname{Fr}\left(f^{j}(C)\right)$ for some $j \geqq 1$; and there could a priori exist other periodic elliptic points associated to some of the components $C_{i}$ (see 10).

14.

Proof of 2. Let $f(z)=z^{m}+a, m \geqq 2, a \in \mathbb{C}$ and we will suppose that we are in the situation of 6 , with $\alpha \in D C$. If we suppose that condition c) in Lemma 13 is true, then we can apply Lemma 13, and using 1 we arrive at a contradiction; hence c) is not true. We deduce from this that $s_{0}=a=f(0) \in \operatorname{Fr}\left(f^{j+1}(C)\right)$ for some $j \geqq 0$. But since $f: \operatorname{Fr}\left(f^{j}(C)\right) \rightarrow \operatorname{Fr}\left(f^{j+1}(C)\right)$ is surjective, this implies $0 \in \operatorname{Fr}\left(f^{j}(C)\right)$ for some integer $j$.

15.

Other Examples. i) Let $f$ be polynomial such that all the critical points except one belong to the basin of attraction of $\infty$. We suppose furthermore $f$ has a periodic elliptic point $z_{0}$ as in 6 , with $\alpha \in D C$. Then $f^{q}$ has a critical value on $\operatorname{Fr}(C)$.

The proof is the same as above.

ii) Let $f(z)=\frac{\lambda_{1} z+\lambda_{2} z^{2}}{(1+z)}, \lambda_{1}=e^{2 \pi i \alpha}, \alpha \in D C$, and $\left|\lambda_{2}\right|>1$ or $\lambda_{2}=1$. Let $C$ be the singular disk associated to $z_{0}=0$. Then $f$ has a critical value on $\operatorname{Fr}(C)$ [this follows by similar arguments to 14 using that basin $B$ of attraction of $\infty$ contains a critical point of $f$ and $\left.f^{ \pm 1}(B)=B\right]$. 
iii) Let $f(z)=e^{2 \pi i \alpha} z+z^{d}, d \geqq 2, \alpha \in D C$. Let $C$ be the disk associated to $z_{0}=0$. Then there exists a critical value of $f$ in $\operatorname{Fr}(C)$. (The proof is similar to the one of 14 by using the fact that $f$ commutes with $z \rightarrow e^{2 \pi i /(a-1)} z$ and by proving an appropriate modification of Lemma 13.)

16.

Proof of 4. Let $f(z)=e^{a z}, a \neq 0, f$ has only one singular value $s_{0}=0$, the omitted value.

We suppose that $f$ has a periodic elliptic point $z_{0}$ with the conditions of 6 (i.e. $C$ has compact closure in $\mathbb{C}$ ) and furthermore $\alpha \in D C$. We want to show that $C$ cannot have compact closure in $\mathbb{C}$. By 3 , it is enough to see that we can apply Lemma 13. This is the case, since all the conditions of Lemma 13 are verified (even c)): for if $0 \in \operatorname{Fr}\left(f^{j+1}(C)\right)$, for some $j \in \mathbb{N}$, this would contradict the fact that $f: \operatorname{Fr}\left(f^{j}(C)\right)$ $\rightarrow \operatorname{Fr}\left(f^{j+1}(C)\right)$ is surjective [by the same proof as for Lemma 1, using that $f^{j}(C)$ has compact closure in $\mathbb{C}]$. By the absurd, 4 follows.

17.

Let us give 2 propositions related to the question of the injectivity of $g=f^{q}$ on $\bar{C}$ (we make the hypotheses of 6).

Proposition 1. If $g$ is a polynomial with no critical values on $\operatorname{Fr}(C)$ then $g$ is injective on the set $A$ of points of $\operatorname{Fr}(C)$ that are accessible by a Jordan arc in $C$.

Proof. Let $x \neq y, x$, and $y \in A$, and $g(x)=g(y)$. Then there exists a Jordan curve $c$, $c=\tilde{c}([0,1])$, where $\tilde{c}:[0,1] \rightarrow \mathbb{C}$ is continuous, injective on $[0,1[, \tilde{c}(0)=\tilde{c}(1)=g(x)$, and $\tilde{c}(] 0,1[) \subset C$.

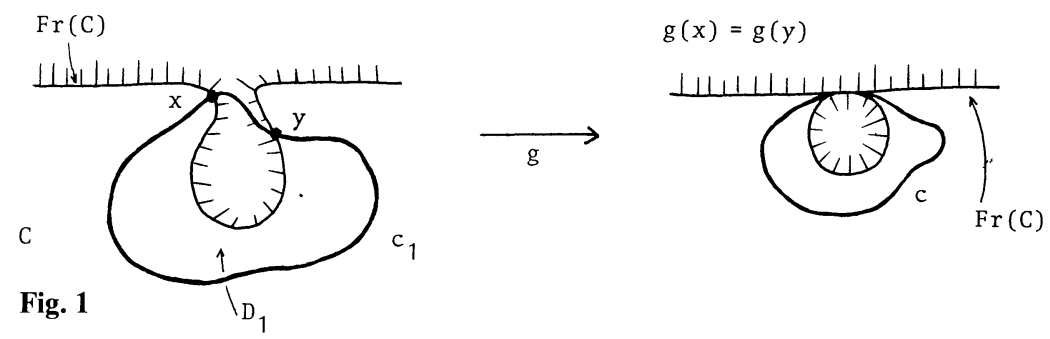

As $g$ is a polynomial and $S(g) \cap \operatorname{Fr}(C)=\emptyset$, by changing the Jordan curve $c$ we can suppose that $c \cap S(g)=\emptyset$ and therefore $g^{-1}(c)$ is a union of closed Jordan curves. It follows that if $c_{1}$ is the closed Jordan curve containing $x, y$, and $\left(g_{\mid C}\right)^{-1}(\tilde{c}(] 0,1[))$, and if $D_{1}$ is the open disk bounded in $\mathbb{C}$ by the Jordan curve $c_{1}$, then by the maximum principle, $\left(g_{\mid D_{1}}^{n}\right)_{n \geqq 0}$ is a normal family since $x \neq y$, $D_{1} \cap J(g) \neq \emptyset$, and the proposition follows since we arrived at a contradiction.

Proposition 2. The map $g_{\mid \mathrm{Fr}(C)}$ has a dense orbit.

Proof. Let $h: \mathbb{D} \rightarrow C$ be the unique conformal map with $h(0)=z_{0}$ and $h^{\prime}(0)>0$. 
We have:

$$
g \circ h(z)=h(\lambda z), \text { for }|z|<1 \text { and } \lambda=e^{2 \pi i \alpha} .
$$

Let $B$ be the set $\left\{z \in \mathbb{S}_{1} \mid \lim _{r \rightarrow 1} h(r z), 0 \leqq r<1\right.$, exists $\}$. By (1) we have for $z \in B, \lambda z \in B$. For $z \in B$ we define $h_{1}(z)=\lim _{r \rightarrow 1} h(r z)$. The set $B$ is a borel subset of $\operatorname{Fr}(C)$ and the mapping $h_{1}: B \rightarrow F(C) \subset \mathbb{C}$ is borel.

Using (1), we obtain:

$$
g \circ h_{1}(z)=h_{1}(\lambda z), \text { for } z \in B .
$$

By Fatou's theorem, the Haar measure of $B$ is equal to 1 . Let $\mu_{1}=d \theta_{\mid B}$ be the induced probability measure on $B$ by the Haar measure of $\mathbb{S}_{1}$, $d \theta$. Let $\mu$ be the probability measure direct image of $\mu_{1}$ by $h_{1}$ [i.e. for every borel subset of $\mathbb{C}, \mu(A)$ $\left.=\mu_{1}\left(h_{1}^{-1}(A)\right)\right]$. By (2), the measure $\mu$ is invariant by $g$ and $g$ is $\mu$-ergodic, since $g$ is a factor of $z \in B \rightarrow \lambda z \in B$. We want to show that the support $\mu$ equals $\operatorname{Fr}(C)$. Let us argue by contradiction: we suppose that there exists $a \in \operatorname{Fr}(C)$ and $\varepsilon>0$, such that the Haar measure of the set

$$
\left\{z \in \mathbb{S}_{1}|| h_{1}(z)-a \mid<\varepsilon\right\}
$$

is equal to 0 .

We consider a Möbius transformation $l(z)=\frac{z-z_{0}}{z-a}$. The holomorphic function $l \circ h: \mathbb{D} \rightarrow \mathbb{C}$ is univalent and therefore by [Du, p. 50], $l \circ h \in H^{p}(\mathbb{D}), 0<p<\frac{1}{2}$, where $H^{p}$ denotes the Hardy space. Our hypothesis implies that $l \circ h_{1} \in L^{\infty}\left(\mathbb{S}_{1}\right)$. Since $l \circ h \in H^{p}, 0<p<\frac{1}{2}$, and $l \circ h_{1} \in L^{\infty}$, we conclude that $l \circ h \in H^{\infty}(\mathbb{D})$ and this contradicts the fact that $\infty \in \operatorname{Fr}(l \circ h(\mathbb{D}))$. By the absurd this proves that the support of $\mu$ equals $\operatorname{Fr}(C)$; and since $g$ is $\mu$-ergodic, Proposition 2 follows.

\section{Appendix. Iteration of Entire Functions}

1.

Let $f: \mathbb{C} \rightarrow \mathbb{C}$ be a holomorphic map (or entire function) and we will suppose in the following that $f$ is not a polynomial of degree 0 or 1 .

By [F], the set $\left(f^{-n}(x)\right)_{n \geqq 1}$ is infinite if $x$ is not the exceptional Fatou value of $f$ [i.e. $x=e_{0} \in \mathbb{C}, n \in \mathbb{N}$, and $f(x)=e_{0}+\left(x-e_{0}\right)^{n} e^{G(x)}, G$ being an entire function]. If $f$ has a omitted value $w$ [i.e. $f(z)=w+e^{G(z)}$, we will denote this value by $w$ with the convention that if $w$ does not exist then $\{w\}=\emptyset$.

2.

We denote the Fatou-Julia set of $f$ by $J(f) \subset \mathbb{C}: x \notin J(f)$, if there is an open set $U \ni x$, such that the family $\left(\left.f^{n}\right|_{U}\right)_{n \geqq 1}$ is a normal family from $U$ into $\mathbb{S}^{2}$ (we allow $\infty$ as a possible limit function). 
We have the following properties:

a) The set $J(f)$ is non empty, closed in $\mathbb{C}$, perfect and, if $J(f) \neq \mathbb{C}$, then $J(f)$ is nowhere dense,

b) $J\left(f^{n}\right)=J(f), n \geqq 1$.

c) $f(J(f))=J(f)-\{w\}, f^{-1}(J(f))=J(f)$ and therefore $f(\mathbb{C}-J(f))=\mathbb{C}-J(f)-\{w\}, f^{-1}(\mathbb{C}-J(f))=\mathbb{C}-J(f)$.

d) As, if $x \in J(f)$, then for every neighborhood $V \ni x, \bigcup_{n \geq 1} f^{n}(V) \supset \mathbb{C}-\left\{e_{0}\right\}, e_{0}$ being, if it exists, the exceptional Fatou value of $f$; it follows that:

For every $x \in J(f)-\left\{e_{0}\right\}$, the set $\left(f^{-n}(x)\right)_{n \geq 1}$ is dense in $J(f)$.

There exists a dense $G_{\delta}, G \subset J(f)$ such that, if $x \in G$, then $\left\{f^{n}(x), n \geqq 1\right\}$ is dense in $J(f)$.

All the above properties are due to Fatou [F] (see [3], [BR], and [6] for the case of rational functions). The following property is due to Baker $\left[\mathrm{B}_{1}\right]$ :

e) The set of repulsive periodic points is dense in $J(f)$.

3.

Remark. Let $x_{0}$ be a periodic point of $f\left[\right.$ i.e. $\left.f^{k}\left(x_{0}\right)=x_{0}\right]$ with multiplier $\left(f^{k}\right)^{\prime}\left(x_{0}\right)$ a root of unity, then $x_{0} \in J(f)$ [if $x_{0} \notin J(f)$, then $f^{k}$ would be periodic and therefore $f$ would be a polynomial of degree 1].

\section{Singular Values of $f\left(=\right.$ Critical Points of $\left.f^{-1}\right)$ (see $\left.\left[\mathrm{B}_{3}\right]\right)$}

They are the values $S(f)$ that stop $f$ from being a covering map (or revêtement in French) (see [A, Chap. I, No. 14, p. 29]) and $S(f)$ is composed of i) and ii):

i) Every algebraic critical value belongs to $S(f)$ [i.e. $f(c) \in S(f)$ with $c \in \mathbb{C}$ and $\left.f^{\prime}(c)=0\right]$.

ii) Every transcendental critical value $s$ (or finite asymptotic value) belongs to $S(f)$ [i.e. there exists a path ${ }^{4} \gamma:[0,1[\rightarrow \mathbb{C}$, such that $\gamma(t) \rightarrow \infty$ and $f(\gamma(t)) \rightarrow s \in \mathbb{C}$, if $t \rightarrow 1]$.

If $f$ has an omitted value $w$ then $w \in S(f)$ (since $w$ is a finite asymptotic value) and if $f$ is a transcendental entire function "one should add" $\{\infty\}$ to $S(f)$ and $J(f)$ (which we will not do).

5.

If $D=\left\{z|| z-z_{0} \mid<r\right\}$ and if $S(f) \cap D=\emptyset$, then $f: f^{-1}(D) \rightarrow D$ is a (trivial) covering (cf. [A, p. 29]).

Let $U$ be open and connected, with $f(U)=U-\{w\}$ and $f(\operatorname{Fr}(U)) \subset \operatorname{Fr}(U)$, where $\operatorname{Fr}(U)$ is the frontier of $U$ in $\mathbb{C}$, then $f_{\mid U}: U \rightarrow U$ is not a covering if one of the following conditions is satisfied (cf. [A, Chap. I, No. 14, p. 291]):

- There exists $c \in U$, a critical point [i.e. $\left.f^{\prime}(c)=0\right]$,

- $\{w\} \neq \emptyset$ and $w \in U$.

- There exists an asymptotic value in $U$ : there exists a path $\gamma(t):[0,1[\rightarrow U$ such that $\gamma(t) \rightarrow \infty$ and $f(\gamma(t)) \rightarrow s \in U$, if $t \rightarrow 1$.

4 We can replace path by Jordan path 
One shows (see $\left[\mathrm{B}_{3}\right]$ ) that the singular values of $f^{n}, n \geqq 1$ are contained ${ }^{5}$ in $\bigcup_{0 \leqq j \leqq n-1} f^{j}(S(f))$ [and the inclusion is an equality if $S(f)$ is bounded].

(We use the following fact: if $f$ and $g$ are non-constant entire functions, then $\left[\mathrm{B}_{3}\right]$

$$
S(f \circ g) \subset S(f) \cup f(S(g)),
$$

and the inclusion is an equality if, for example, $S(g)$ is bounded since

$$
g: g^{-1}(\mathbb{C}-\overline{S(g))} \rightarrow \mathbb{C}-\overline{S(g)}
$$

is a covering map.)

\section{We suppose that $J(f) \neq \mathbb{C}$}

Let $C$ be a component of $\mathbb{C}-J(f)$. Then there are only the 2 possibilities (considered by Fatou [3] and Julia):

a) The sequence $\left(\left.f^{n}\right|_{c}\right)_{n \geqq 0}$ has only constant limit functions [a limit function means the limit in the compact open topology of a converging subsequence $\left(\left.f^{n_{i}}\right|_{C}\right)_{i \geqq 0}$ with $\left.0<n_{i}<n_{i+1}\right]$.

b) There exists a non-constant limit function.

There are also the 2 possibilities:

1) $C$ is wandering [i.e. $\left(f^{n}(C)\right)_{n \geqq 0}$ are mutually disjoint].

2) $C$ is preperiodic [i.e. there exists integer $k \geqq 0$ and $q \geqq 0$, such that $\left.f^{k+q}(C)=f^{k}(C)\right]$.

Fatou [3, Sect. 28] (see also [BR]) shows that 1) implies a).

To study the case 2), it is enough to suppose that $f(C)=C-\{w\}$. We have then the possibilities a) and b). We will divide a) into:

$\left.\mathrm{a}_{1}\right)$ The sequence $\left(f_{\mid C}^{n}\right)_{n \geqq 1}$ has one finite constant limit function and $C$ is called a finite Fatou component.

$\left.\mathrm{a}_{2}\right)$ The sequence $\left(f_{\mid C}^{n}\right)_{n \geqq 1}$ tends to $\infty$ and $C$ is called an infinite Fatou component.

\section{Wandering Domains}

Baker showed in $\left[\mathrm{B}_{4}\right]$ that wandering domains could exist for an entire transcendental function. For other examples, see [6, II.11]. All the known examples satisfy: $\left(\left.f^{n}\right|_{C}\right)_{n \geqq 1}$ tends to $\infty$.

Question. Does there exist an entire function $f$ with a wandering component $C$ of $\mathbb{C}-J(f)$ such that $\bigcup_{n \geqq 0} f^{n}(C)$ has compact closure in $\mathbb{C}$ ? (If $C$ exists, by the maximum modulus principle, $C$ is simply connected.)

Sullivan showed that for a rational function $f$ of $\mathbb{S}^{2}$ every component $C$ of $\mathbf{S}^{2}-J(f)$ [we suppose that $\mathbf{S}^{2}-J(f)$ is non-empty] is preperiodic (see [S]). Baker showed $\left[\mathrm{B}_{5}\right]$ that for every entire function of the form

$$
f(z)=\int_{z_{0}}^{z} P(u) e^{Q(u)} d u \quad \text { or } \quad f(z)=P_{1}\left(e^{z}\right)
$$

5 Baker showed that one can replace contained in by equal to 
where $P$ and $Q$ are polynomials and $P_{1}$ is a non-constant polynomial, then every component of $\mathbb{C}-J(f)$ is non-wandering (and therefore is preperiodic).

In particular this is the case for $f(z)=e^{a z}, a \in \mathbb{C}-\{0\}$ (this special case follows also from $[\mathrm{G}]$ and $\left[\mathrm{B}_{5}\right.$, Sect. 3$]$ ).

\section{Finite Fatou Components $C, f(C)=C-\{w\}$}

Fatou shows that $\left(\left.f^{n}\right|_{C}\right)_{n \geqq 1}$ tends locally uniformly on $C$ to $x_{0} \in \bar{C}, x_{0} \in \mathbb{C}$, and $f\left(x_{0}\right)=x_{0}[3$, Sect. 56] (what is used is that the fixed points of $f$ are discrete in $\mathbb{C}$ and $f$ is holomorphic on $\left.\mathbb{S}^{2}-\{\infty\}\right)$.

If $x \in C$, then $m=f^{\prime}\left(x_{0}\right)$ (the multiplier) satisfies $|m|<1$.

If $x \notin C$, then $m=1$ [3, Sect. 54].

Furthermore, every fixed point $x_{0}$ of $f$, with $|m|<1$ gives rise to one finite Fatou component and every fixed point $x_{0}$ of $f$ with $m=1$ gives rise to $d-1$ finite Fatou components, where $d$ is the order of contact $f$ to the identity at $x_{0}$ [3, Sect. 9 and Sect. 11].

Furthermore, Fatou shows $\left[3\right.$, Sect. 30] that $f_{\mid C}: C \rightarrow C$ can not be a covering map, and it follows that $C \cap S(f) \neq \emptyset$.

\section{Infinite Fatou Components, $C, f(C)=C-\{w\}$}

Then $\left(\left.f^{n}\right|_{C}\right)_{n \geqq 1}$ tends locally uniformly to $\infty$. Baker showed that $C$ is simply connected $\left[\mathrm{B}_{2}\right]$. There are the 2 possibilities:

1) $\left.f\right|_{C}: C \rightarrow C$ is not a covering map. The first example $z \rightarrow z+1+e^{-z}$ was given by Fatou [F, Sect. 15].

2) $\left.f\right|_{C}$ is a $\mathbb{C}$-diffeomorphism. (It is not difficult to see that this implies that $f$ has an infinite dimensional space of quasiconformal deformations.)

Examples. i) Let $a \in \mathbb{R}, 0<a<1, b \in \mathbb{R}$, and $f_{b}(z)=z+\frac{a}{2 \pi} \sin (2 \pi z)+b$. If we choose the number $b$ such that the rotation number of $f_{b}, \varrho\left(f_{b \mid \mathbb{R}}\right)=\alpha \in \mathbb{R}$, satisfies $\alpha \in D C$ (which is possible by [5, II and III]), then we can use I.3 and complexification to see that $f$ leaves invariant an infinite Fatou component $C \supset \mathbb{R}$. Let us show that $f_{\mid C}$ is a $\mathbb{C}$-diffeomorphism. We denote by $p: \mathbb{C} \rightarrow \mathbb{C} / \mathbb{Z}$, the canonical projection, and set $R_{q}(z)=z+q, q \in \mathbb{Z}$. Then, since $R_{q} \circ f=f \circ R_{q}$, one deduces that $R_{q}(C)=C$. We denote by $\bar{f}: \mathbb{C} / \mathbb{Z} \rightarrow \mathbb{C} / \mathbb{Z}$ the induced map by the entire function $f$. We have: $\bar{f}(p(C))=p(C)$. It follows that the family $\left(\bar{f}_{\mid p(C)}^{n}\right)_{n \geqq 1}$ is normal (since it omits more than 2 values of $\mathbb{C}^{*}$ ) with non-constant limit functions (since $\bar{f}_{\mid \mathbb{R} / \mathbb{Z}}$ is of degree 1 ). Therefore $p(C) \subset \mathbb{R} / \mathbb{Z}$ is contained in (and is even equal to) a singular domain of $\bar{f}$; and by the same argument as in [3, Sect. 28] (see also [BR]), we conclude that $p(C)$ is a ring (or a disk) and that $\bar{f}_{\mid p(\boldsymbol{C})}$ is a $\mathbb{C}$-diffeomorphism.

It follows that $f_{\mid C}$ is a $\mathbb{C}$-diffeomorphism $[p(C)$ is a ring since $C$ is connected and it contains $\mathbb{R}]$.

ii) Let $\alpha \in D C$,

$$
f(z)=z+2 \pi i \alpha+e^{z}
$$

and

$$
g(z)=e^{2 \pi i \alpha} z e^{z}
$$


One has, for $q \in \mathbb{Z}, R_{q} \circ f=f \circ R_{q}$ and a commutative diagram:

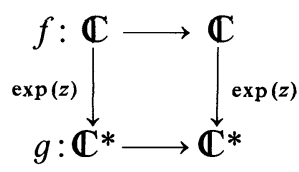

One concludes, using Siegel's theorem [9] (see also [6, VIII.6]) applied to $g$ at $z=0$, that $f$ has an infinite invariant Fatou component, $C$, containing $\{\operatorname{Re} z<-k\}$ with $k$ a large positive real number. In a similar way to i), one shows that $f_{\mid C}$ is a $\mathbb{C}$-diffeomorphism.

iii) To give an example of 1), it is enough to take in the example of ii), $\alpha=p_{1} / q_{1} \in \mathbb{Q}$, use the fact that $g$ has at least $q_{1}-1$ finite Fatou components at 0 (with 12), and then replace, if necessary, $\alpha$ by $\alpha+p_{2}, p_{2} \in \mathbb{Z}$, (to avoid wandering domains). (Fatou's example is a special case of the case when $g$ has an attracting fixed point at 0 .)

\section{Singular Components $C, f(C)=C$}

If $\left(f_{\mid C}^{n}\right)_{n \geqq 1}$ has a non-constant limit function, then $f_{\mid C}$ is a $\mathbb{C}$-diffeomorphism and the closure in the compact open topology (on $C$ ) of the sequence $\left(f^{n} \mid{ }_{C}\right)_{n \in \mathbb{Z}}$ is isomorphic to the circle group $\mathbb{S}_{1}$ (see $[3$, Sect. 28] or [BR] and also $[6, \mathrm{I}]$ ).

Since an entire function $C$ cannot have a ring, $C$ is $\mathbb{C}$-diffeomorphic to $\mathbb{D}$ and $f$ has a fixed point $z_{0} \in C$ with

$$
f^{\prime}\left(z_{0}\right)=e^{2 \pi i \alpha}, \quad \alpha \in \mathbb{R}-\mathbb{Q} .
$$

If $h: \mathbb{D} \rightarrow C$ is a $\mathbb{C}$-diffeomorphism, with $h(0)=z_{0}$, one has, for $|z|<1$,

$$
h^{-1} \circ f \circ h(z)=e^{2 \pi i \alpha} z .
$$

By the same argument as for rational functions [3, Sect. 30] or [BR],

$$
\overline{\bigcup_{0 \geqq j} f^{j}(S(f))} \text { contains } \operatorname{Fr}(C) \text {. }
$$

\section{Cases where $\mathbb{C}=J(f)$}

Baker gave in $1970\left[\mathbf{B}_{3}\right]$, an example of an entire function $f$ such that $J(f)=\mathbb{C}$, and Misiurewicz showed this for $z \rightarrow e^{z}[\mathrm{M}]$. Baker and Rippon showed in [B $\mathrm{B}_{6}$, that there exists a dense $G_{\delta^{-}}$-set, $G \subset \mathbb{T}^{1}$, such that, if $\alpha \in G$, then the entire function

$$
f: z \rightarrow e^{2 \pi i \alpha}\left(e^{z}-1\right)
$$

satisfies $J(f)=\mathbb{C}$.

Let us notice that by 2.d) and the description of the components given from 6 to 10, we have:

- $J(f)=\mathbb{C}$.

企

- There exists $z \in \mathbb{C}$ such that the orbit $\left(f^{n}(z)\right)_{n \geqq 0}$ is dense in $\mathbb{C}$.

Remark. Let $f(z)=z+\ldots$ be an entire function different from the identity. For 
$\alpha \in \mathbb{T}^{1}$, let $f_{\alpha}(z)=e^{2 \pi i \alpha} f(z)$. Then there exists a dense $G_{\delta}$-set, $G \subset \mathbb{T}^{1}$, such that, if $\alpha \in G$, then $f_{\alpha}$ is not linearizable at 0 . To show this, let:

$$
\left.(\alpha, r) \in \mathbb{T}^{1} \times\right] 0,+\infty\left[\rightarrow \varphi(\alpha, r)=\sup _{n \geqq 0} \sup _{|z|=r}\left|f_{\alpha}^{n}(z)\right| \in \mathbb{R} \cup\{+\infty\} .\right.
$$

For $\alpha \in \mathbb{T}^{1}$ fixed, $r \rightarrow \varphi(\alpha, r)$ is nondecreasing by maximum modulus principle and for $r$ fixed, $\alpha \rightarrow \varphi(\alpha, r)$ is lower semicontinuous.

$$
\begin{gathered}
\text { Let } G_{1 / n}=\left\{\alpha \in \mathbb{T}^{1} \mid \varphi\left(\alpha, \frac{1}{n}\right)=+\infty\right\} \text {. The set } G_{1 / n} \text { is a } G_{\delta} \text { and so is } \\
\qquad \begin{array}{l}
G=\bigcap_{n \geqq 1} G_{1 / n} .
\end{array}
\end{gathered}
$$

To see that $G$ is dense, it is enough to remark that $G \supset \mathbb{Q} / \mathbb{Z}$ (cf. 3) and $\alpha \in G$ iff $f_{\alpha}$ is linearizable.

(On related questions the reader should consult Cremer's paper [C].)

\section{Connectivity of Components of $\mathbb{C}-J(f)\left[\mathrm{B}_{5}\right.$, Sect. 3]}

Let $C$ be a component of $\mathbb{C}-J(f)$. If $C$ is not simply connected then:

- $f$ is a polynomial and $C$ is the basin of attraction of $\infty$ [ $C$ has infinite connectivity iff there exists a critical point $c$ of $f$ such that $f^{n}(c) \rightarrow \infty$, if $\left.n \rightarrow+\infty\right]$.

- The component $C$ is a wandering component of $\mathbb{C}-J(f)$ (for an example of a non-simply connected wandering component, see $\left.\left[\mathrm{B}_{4}\right]\right)$.

In all the other cases $C$ is simply connected [if $C$ is a finite preperiodic Fatou component (cf. 8) or a preperiodic singular component (cf. 10) this follows from the maximum modulus principle; for infinite preperiodic Fatou components, this follows from $\left.\left[\mathrm{B}_{2}\right]\right]$.

Remark. If $C$ is a component of $\mathbb{C}-J(f)$ and if the closure $\bar{C}-\{\infty\}$ of $C$ (in $\mathbb{C}$ ) satisfies $\bar{C}-\{\infty\} \neq \mathbb{C}$, then $C$ is the interior (in $\mathbb{C}$ ) of $\bar{C}$ - $\{\infty\}$ [for the family $\left(f_{\mid C}^{n}\right)_{n \geqq 0}$ omits more than 3 values].

For $f(z)=e^{z}-e^{z_{0}}$, where $z_{0} \in \mathbb{C}$ satisfies $\left|e^{z_{0}}\right|<1$, the basin of attraction of $z_{0}, C$, is such that $\bar{C}=\mathbb{S}^{2}$.

\section{References}

1. Ahlfors, L.V.: Lectures on quasiconformal mappings. Princeton: Van Nostrand 1966

2. Carathéodory, C : Theory of functions of a complex variable. Vol. II. New York: Chelsea 1954

3. Fatou, M P : Sur les équations fonctionnelles. Bull Soc. Math. Fr. 47, 161-271 (1919); 48, 33-94, 208-304 (1920)

4. Ghys, E.: Transformation holomorphe au voisinage d'une courbe de Jordan. C.R. Acad. Sc. Paris, t. 289, 385-388 (1984)

5. Herman, M.R.: Sur la conjugaison différentiable des difféomorphismes du cercle à des rotations. Publ. Math. I.H.E.S. 49, 5-233 (1979)

6. Herman, M.R.: Examples de fractions rationnelles ayant une orbite dense sur la sphère de Riemann. Bull. Soc. Math. France 112, 93-142 (1984)

7. Pommerenke, C.: Univalent functions. Göttingen: Vandenhoeck \& Ruprecht 1975

8 Rudin, W.: Real and complex analysis. New York: McGraw-Hill 1974

9. Siegel, C.L.: Iteration of analytic functions Ann. Math 43, 607-612 (1942) 
10. Yoccoz, J.C.: Conjugaison différentiable des difféomorphismes du cercle dont le nombre de rotation véfifie une condition diophantienne. Ann Sci. Ec. Norm Sup., 4ème séries, t. 17, 333-359 (1984)

11. Yoccoz, J.C.: $C^{1}$-conjugaison des difféomorphismes du cercle. Lecture Notes in Mathematics, Vol. 1007, pp. 814-827. Berlin, Heidelberg, New York: Springer 1983

[A] Ahlfors, L.V., Sario, L.: Riemann surfaces. Princeton, NJ: Princeton University Press 1960

$\left[\mathrm{B}_{1}\right]$ Baker, I.N.: Repulsive fixpoints of entire functions. Math. Z. 104, 252-256 (1968)

$\left[\mathrm{B}_{2}\right]$ Baker, I.N.: The domains of normality of an entire function. Ann. Acad. Fennicae, Ser. A I 1, 277-283 (1975)

$\left[\mathrm{B}_{3}\right]$ Baker, I N.: Limit functions and sets of non-normality in iteration theory. Ann. Acad. Sci. Fennicae, Ser. A I 467, 1-11 (1970)

$\left[\mathrm{B}_{4}\right]$ Baker, I.N.: An entire function which has wandering domains. J. Australian Math. Soc. (Ser. A) 22, 173-176 (1976)

$\left[\mathrm{B}_{5}\right]$ Baker, I.N.: Wandering domains in the iteration of entire functions, Report No 20, Institut Mittag-Leffler (1983)

[B $\left.{ }_{6}\right]$ Baker, I.N., Rippon, P.J.: Iteration of exponential functions, Ann. Acad. Sci. Fennicae, Ser. AI 9, 49-77 (1984)

[BJ] Brjuno, A.D.: Analytical form of a differential equation. Trans. Moscow Math. Soc. 25, 131-288 (1971)

[BR] Brolin, H.: Invariant sets under iteration of rational functions. Ark. Mat 6, 103-144 (1966)

[C] Cremer, H.: Über die Häufigkeit der Nichtzentren. Math. Ann. 115, 573-580 (1938)

[D] Douday, A., Hubbard, J.H.: On the dynamics of polynomial like mappings. Ann. Sci. Ec. Nor. Sup. (to appear)

[Du] Duren, P.L.: Theory of $H^{p}$ spaces. New York: Academic Press 1970

[F] Fatou, P.: Sur l'itération des fonctions transcendantes entières. Acta Math. 47, 337-370 (1926)

[G] Ghys, E., Goldberg, L., Sullivan, D.: On the measurable dynamics of $z \rightarrow e^{z}$. Erg. Th. Dyna. Sys. (to appear)

[HY] Hocking, J.G., Young, G.S.: Topology, pp. 143-145. Reading, MA: Addison-Wesley 1961

[M] Misiurewicz, M.: On iterates of $e^{z}$. Erg. Th. Dyna. Syst. 1, 103-106 (1981)

[S] Sullivan, D.: Quasiconformal homeomorphisms and dynamics I, preprint I.H.E.S. (1982). Ann. Math. (to appear)

Communicated by J. Mather

Received October 29, 1984; in revised form January 22, 1985 